\title{
Correlation of Tumor Necrosis Factor Alpha, Interleukin 6 and Interleukin 10 With Blood Pressure, Risk of Preeclampsia and Low Birth Weight in Gestational Diabetes
}

\author{
P. $\check{Z Z A ́ K}^{1}$, M. SOUČEK ${ }^{1}$ \\ ${ }^{1}$ Second Department of Internal Medicine, Faculty of Medicine, Masaryk University, Brno, Czech \\ Republic
}

Received August 16, 2018

Accepted January 14, 2019

Epub Ahead of Print March 22, 2019

\section{Summary}

Several pathophysiological mechanisms have been proposed in the development of pregnancy complications, including endothelial dysfunction, an inflammatory pathway and oxidative stress. The aim of the present study was to evaluate the correlation between proinflammatory cytokines TNF-a, IL- 6 and dual cytokine IL-10 in the mother's peripheral blood and systolic blood pressure, risk of preeclampsia and low birth weight in gestational diabetes (GDM). We observed 40 women with GDM divided into a gestational hypertension group $(n=20)$ and comparison group $(n=20)$ with normal blood pressure. We found a significant positive correlation between TNF- $a$; IL-6; IL-10 levels and systolic blood pressure (SBP) in the second trimester $(p<0.001 ; p<0.001 ; p<0.001)$; the third trimester $(p<0.001$; $p<0.001 ; p<0.05)$. We also proved correlations for diastolic blood pressure (DBP) during the second; third trimester $(p<0.001$; $\mathrm{p}<0.001 ; \quad \mathrm{p}<0.001) ; \quad(\mathrm{p}<0.001 ; \quad \mathrm{p}<0.001 ; \quad \mathrm{p}<0.0015)$. We demonstrated a statistically significant positive association between high TNF-a group and preeclampsia risk in the third trimester $(p=0.04)$. We also determined the negative correlation in the second trimester between birth weight and TNF-a; IL-6, IL-10 levels ( $p<0.05 ; p<0.001 ; p<0.001)$. To conclude, our data highlight the importance of cytokines TNF-a, IL- 6 and IL-10 in blood pressure regulation. In addition, high levels of TNF-a have been associated with increased risk of preeclampsia. We found a significant negative correlation between levels of TNF-a, IL-6, IL-10 and birth weight.

\section{Key words}

Hypertension • Preeclampsia • Interleukin 6 • Tumor necrosis factor alpha $\bullet$ Low birth weight

\section{Corresponding author}

P. Zak, Second Department of Internal Medicine, Faculty of Medicine, Masaryk University, Pekarska 53, 65691 Brno, Czech Republic. E-mail: petr.zak@fnusa.cz

\section{Introduction}

Gestational diabetes mellitus (GDM) is defined as diabetes diagnosed in the second or third trimester of pregnancy that was not clearly overt diabetes prior to gestation. Approximately $7 \%$ of all pregnancies are complicated by GDM. The prevalence may range from 1 to $14 \%$ of all pregnancies, depending on the population studied. Types 1 and 2 pregestational diabetes confer significantly greater maternal and fetal risk than GDM. In general, specific risks of uncontrolled diabetes in pregnancy include spontaneous abortion, fetal anomalies, preeclampsia, fetal demise, macrosomia, neonatal hypoglycemia, and neonatal hyperbilirubinemia, among others (ADA Standards of Diabetes Care 2018). As early as 1995 announced the original Th1/Th2 paradigm is based on Wegmann's statement: 'successful pregnancy is a Th2 phenomenon'. In pregnancy Th1-associated cytokines tumor necrosis factor alpha (TNF)- $\alpha$ and interferon gamma and in general induction of an inflammatory response were associated with fetal demise (Chaouat et al. 2004). The production of Th2-type cytokines IL-4 and IL-10 may promote fetal survival by inhibiting Th1 reactivity (Azizieh et al. 2015). Many studies have reported a predominant Th2-type immunity and suppressed Th1-type immunity during normal 
pregnancy. In a normal pregnancy the levels of cytokines in the mother's serum are not always detectable. Elevated levels of cytokines in the mother's peripheral blood can initiate a cascade of inflammatory processes and lead to complications in a so far normal pregnancy (Lash et al. 2015). Elevated levels of cytokines can be the cause of placental dysfunction, which may result in intrauterine growth restriction (IUGR) or premature birth (PTB) (Challis et al. 2009). De Steenwinkel et al. (2013) proposed a hypothesis, that the levels of maternal cytokines can significantly influence the growth of the fetus in women with rheumatoid arthritis (RA). They proved, that in the mother's serum during the first trimester, increased levels of interleukin 10 (IL-10) are associated with higher birth weight, and increased levels of interleukin 6 (IL-6) are associated with lower birth weight (De Steenwinkel et al. 2013). In studies on pregnant women, it has been proven that both TNF- $\alpha$ and IL-6 induce structural and functional changes of endothelial cells. Endothelial dysfunction is associated with many forms of hypertension and was, in human studies, at least partially mediated by increased levels of proinflammatory cytokines (Saito et al. 2001). Preeclampsia (PE) affects 5 to $8 \%$ of pregnancies and imposes a significant burden on the health care system with high maternal and fetal morbidity, intrauterine growth restriction (IUGR) and preterm birth. Without timely intervention, $\mathrm{PE}$ is conducive of a more grievous clinical picture of maternal seizures and convulsions, which could culminate into coma and death (Shah et al. 2015). An up-regulation of proinflammatory cytokines (TNF- $\alpha$, IL-1 $\beta$, IL-18) was described in pregnancies complicated by PE (Wang et al. 1996), other studies have proved a decrease in levels of anti-inflammatory cytokine IL-10 (Hennessy et al. 1999). Only a small number of studies analyzed the role of cytokines in the pathophysiology of oxidative stress in GDM. In current literature, we will find the cytokine TNF- $\alpha$ to be the most significant predictor of insulin resistance in pregnancy (Kirwan et al. 2002). However, other cytokines such as IL-6, IL-8 also significantly participate in the pathogenesis of insulin resistance in GDM (Gomes et al. 2013). In conclusion, current literature shows the importance of cytokines in the acceleration of proinflammatory state, in both GDM itself, and in the pathogenesis of certain pregnancy complications: hypertension, $\mathrm{PE}$, low birth weight.

The aim of the present study was to evaluate the correlation between proinflammatory cytokines TNF- $\alpha$;
IL-6 and dual cytokine IL-10 in the mother's peripheral blood and the development of pregnancy complications: increase of systolic blood pressure (SBP); diastolic blood pressure (DBP), risk of PE, low birth weight in women with GDM and gestational hypertension (hypertension group), and comparison group with GDM and normal levels of blood pressure (reference group).

\section{Methods}

The study has been approved by an ethics committee of St. Anne's University Hospital in Brno. There were 40 women with GDM included in the observations, divided into two groups, 20 women with GDM and gestational hypertension, and 20 women with GDM and normal blood pressure levels.

GDM criteria were as follows (one or more of the following criteria were met in pregnancy): fasting plasma glucose 5.1-6.9 mmol/l, 1-hour plasma glucose $\geq 10.0 \mathrm{mmol} / 1, \quad$-hour plasma glucose $\geq 8.5 \mathrm{mmol} / 1$ following a $75 \mathrm{~g}$ oral glucose load. These women were monitored at the diabetes center of St. Anne's University Hospital in Brno. All the women involved signed an informed consent before starting the study. To participate, subjects in gestational hypertension group, had to have in documentation new-onset hypertension occurring after 20 weeks' gestation. Blood pressure (BP) values measured at routine clinical visit in a gynecologist's office at 22 weeks' gestation had to be as follows: $\quad \mathrm{SBP} \geq 140 \mathrm{~mm} \mathrm{Hg}$ and $/$ or $\quad \mathrm{DBP} \geq 90 \mathrm{~mm} \mathrm{Hg}$, reference group: women without an adverse obstetric history with normal blood pressure, i.e. lower than hypertension threshold in referred week of gestation. The reference group was chosen to fit the characteristics of the hypertension group in age, BMI and diabetes compensation (HbAlc levels). Subjects were not engaged in the study if at least one of the following criteria were met: chronic hypertension, history of drug treatment of dyslipoproteinemia, chronic diseases of the immune system, neurological disorders, malignancy, women who were smokers before and during their pregnancy. The control group comprised a consecutive sample of pregnant women followed-up at our setting and undergoing routine late second or third-trimester blood analysis and with none of the exclusion criteria. Tight control of glycemia level was maintained throughout study duration using diabetic diet. Since insulin was required to achieve glycemic control, we chose basal insulin to keep fasting glycemia in target range according 
to the established recommendations of the Czech Diabetes Society. BP levels were taken before the peripheral blood examination was carried during the second trimester $\left(26^{\text {th }}\right.$ week) and the third trimester ( $38^{\text {th }}$ week) in a quiet clinic room. For those who were engaged in the study, their BP was measured three times using an Oscillometric BP device (Omron Diagnostics) after an initial five-minute rest period. This typically occurred in a fasting state and in the morning. Three consecutive recordings were made $30 \mathrm{~s}$ apart. In this gestational hypertension group, the condition was treated according to the established recommendations of the Czech Diabetes Society. No adjustments to the treatment of these patients were put in place due to their participation in the trial. Methyldopa (a centrally acting adrenergic antagonist) was the first choice of antihypertensive agent prescribed to treat this condition. Betaxolol (beta-blocker) was a $2^{\text {nd }}$ alternative treatment. The percentages of subjects; average dosages in mg per day were as follows: monotherapy of methyldopa (85; 164), monotherapy of betaxolol $(0 ; 0)$, a combination (betaxolol+methyldopa) $(15 ; 437.50)$. Other classes of antihypertensive drugs were not used.

The peripheral blood examination was carried out from the venous plasma after $8 \mathrm{~h}$ of fasting during the second trimester $\left(26^{\text {th }}\right.$ week) and the third trimester ( $38^{\text {th }}$ week) of pregnancy. From the immunological parameters we monitored the levels of cytokines TNF- $\alpha$, IL-6 and IL-10, T lymphocyte and NK cells surface antigen expression, absolute count of $\mathrm{CD}(4)+$ cells. To assess the Th1/Th2 immunological profile ratio we chose ratio of Th1 cytokine IL-6/Th2 cytokine IL-10. To evaluate activation of cell-mediated immunity we chose absolute counts of CD4(+) T cells, from the metabolic parameters we monitored glycemia, glycated hemoglobin Alc (HbA1c), plasma uric acid (UA), immunoreactive insulin (IRI) and basic biochemical parameters. After centrifugation, all samples were frozen at $-80{ }^{\circ} \mathrm{C}$ until assayed. Serum levels of IL-6, IL-10 and TNF $\alpha$ were determined using Sandwich ELISA, Biotin-labelled antibody kits (Biovendor Research and Diagnostic Products): HUMAN INTERLEUKIN-6 high sensitivity ELISA, HUMAN INTERLEUKIN-10 high sensitivity ELISA, TNF-alpha Human High Sensitivity ELISA. The lower limit of quantification was IL-10 1.0; IL-6 1.0; TNF- $\alpha$ 4.0. All cytokine levels are presented in $\mathrm{pg} / \mathrm{ml}$. Clinical characteristics were collected by medical record and physical examination. They included maternal age and gynecological history. From the acquired readings we calculated hepatic IR using the equation of Matthews et al. $(1985)$ (HOMA-IR $=($ FPI x FPG)/22.5), where FPI stands for fasting plasma insulinemia (mU/1), FPG for fasting plasma glycemia (mmol/l) (Matsuda et al. 1999). Preeclampsia has been defined as blood pressure: $140 \mathrm{~mm} \mathrm{Hg}$ or higher SBP or $90 \mathrm{~mm} \mathrm{Hg}$ or higher DBP after 20 weeks of gestation and proteinuria of at least $300 \mathrm{mg}$ in a 24-hour urine collection. Gestational hypertension was defined as new-onset hypertension ( $\mathrm{SBP} \geq 140 \mathrm{~mm} \mathrm{Hg}$ and/or $\mathrm{DBP} \geq 90 \mathrm{~mm} \mathrm{Hg}$ ), occurring after 20 weeks' gestation (Wagner et al. 2004). Low birth weight (LBW) was defined by the W.H.O. criteria as a birth weight of less than $2,500 \mathrm{~g}$ (up to and including $2,499 \mathrm{~g}$ ), the birth weight of an infant was the first weight recorded after birth. W.H.O. Infant Weight for Age Percentiles (newborns) calculation was used to calculate weight-for-age percentiles. Preterm infants are those born at $<37$ week from first day of the last menstrual period, regardless of birth weight (Cutland et al. 2017). The observed parameters to be considered at the onset of labor include: week of gestation, mode of delivery (natural; cesarean section), labor induction, other labor complications.

\section{Statistics}

In terms of quantity, descriptive statistics of a sample includes: mean, median, standard deviation, min and max. For categorical variables, absolute frequencies (N) and relative frequencies (\%) are calculated. Comparison of means (SBP; DBP; birth weight; absolute CD4(+) count) obtained in the second and third trimester on reference group and gestational hypertension group was done using Student t-test. The values of TNF- $\alpha$, IL-10, IL-6 and UA were divided according to its median in a group with values lower than the median "low" and higher than the median "high". After completing the analysis of the acquired data, we could state that the sample was equally divided. When the cytokine level was not in sample measurable, the median of the detectable cytokine levels was taken. The Kruskal-Wallis test was chosen to analyze the difference between the groups "low" and "high" in SBP. It is a non-parametric method of testing that doesn't assume normal distribution, therefore being suitable for the comparison of two small sample groups, which corresponded the size of our analyzed sample, p-value is the resultant. A Chi-square test was chosen to evaluate the relationship between SBP and PE risk in a group low; high for IL-6; TNF- $\alpha$; IL-10 cytokines (the Chi-square test is intended to test if there is 
statistically significant difference in terms of frequencies between two categories). To evaluate the correlation between cytokine levels IL-6; TNF- $\alpha$; IL-10 and birth weight; systolic blood pressure SBP; diastolic blood pressure DBP variables, correlation matrix, that shows Spearman's rho correlation, was chosen. The Spearman's rank correlation coefficient, is a non-parametric measure of relation between variables using ranks to calculate the correlation. Sometimes, Spearman's correlation is defined as Pearson's correlation coefficient between rank variables. While Pearson's correlation describes how well relationship between variables can be described using linear function, Spearman's correlation assesses how well the relationship between variables can be described using a monotonic function. Statistically significant correlations are marked bold.

\section{Results}

The descriptive statistics of acquired readings is shown in Table 1. To assess the level of glycemic control achieved by each group we chose measurement of HbAlc level. There was no statistically significant difference in diabetes compensation (HbAlc levels) between hypertension and reference group in the second; third semester $(p=0.78 ; p=0.75)$ (Table 2 ). In the second and third trimester we proved a statistically significant difference in SBP (Fig. 1); DBP (Fig. 2) between hypertension and reference group $(\mathrm{p}<0.001 ; \mathrm{p}<0.001)$; $(\mathrm{p}<0.001 ; \mathrm{p}<0.001)$. An interesting situation, regarding levels of IL- 6 , occurred after analyzing the acquired data. The median was the same as the minimum (more than $50 \%$ of the observed were equal to minimum i.e. 0.5). Therefore, we divided the observed sample of IL- 6 into "low IL-6" for values up to 0.5 and inclusive and "high IL-6" for values above 0.5. Analogically, we used the same method for tracked levels of TNF- $\alpha$, IL-10 and uric acid, dividing them according to the median into a group with a lower value than the median "low" and a higher than the median "high". For the analysis between groups "low" and "high" in SBP, we chose the Kruskal-Wallis test. The analysis' outcome of the mean SBP value in second and third trimester for groups low IL-6; high IL-6 (Table 3) Fig. 1, groups low TNF- $\alpha$; high TNF- $\alpha$ (Table 3) Fig. 2, groups low IL-10; high IL-10 (Table 3) Fig. 3. The relation was evaluated at a $5 \%$ level of significance, we proved a statistically significant difference in IL-6 $(\mathrm{p}<0.001$; $\mathrm{p}<0.001), \quad$ TNF- $\alpha \quad(\mathrm{p}<0.001 ; \mathrm{p}<0.001), \quad$ IL-10 $\quad(\mathrm{p}<0.001 ;$ $\mathrm{p}=0.0019)$ (Tables 2, 3). After using the correlation matrix
(Spearman correlation rho), we analyzed the correlation between IL-6; TNF- $\alpha$; IL-10 cytokine levels and SBP. We found a significant correlation between SBP and IL-6; TNF- $\alpha$ IL-10 levels in the second trimester $0.84 ; 0.55 ; 0.81$ $(\mathrm{p}<0.001 ; \mathrm{p}<0.001 ; \mathrm{p}<0.001$; the third trimester $0.85 ; 0.75$; $0.45, \quad(\mathrm{p}<0.001 ; \mathrm{p}<0.001 ; \mathrm{p}<0.05)$. We also proved correlations for DBP during the second; third trimester $0.79 ; 0.52 ; 0.76(\mathrm{p}<0.001 ; \mathrm{p}<0.001 ; \mathrm{p}<0.001) ; 0.87 ; 0.70$; $0.49(\mathrm{p}<0.001 ; \mathrm{p}<0.001 ; \mathrm{p}<0.0015)$ (Table 4). Using the Chi-square method of testing, we analyzed the relation between the cytokine levels and the risk of development of pregnancy complications. Where we proved a statistically significant relation between high TNF- $\alpha$ group and PE risk in the third trimester $(p=0.04)$, without the proof of statistically significant association for cytokine levels IL-6; IL-10 (Table 5). In the second trimester, the increase of PE risk didn't reach a statistical significance for any of the monitored cytokines (data not shown).

We found the statistically significant difference in the birth weight $(p<0.05)$ (Fig. 3), while we did not prove statistically significant difference in the weight-forage percentiles $(p=0.17)$ between hypertension and reference group. The evaluation of correlation coefficient for birth weight during the second trimester, proved statistically significant negative correlations for all the monitored cytokine levels TNF- $\alpha$; IL-10; IL-6 (-0.32; $-0.51 ;-0.56)$, $p$-values $(\mathrm{p}<0.05 ; \mathrm{p}<0.001 ; \mathrm{p}<0.001)$. In the third trimester, there were also negative correlations $(-0.53 ;-0.27 ; \quad-0.51), \quad p$-values $(\mathrm{p}<0.05 ; \mathrm{p}=0.096$; $\mathrm{p}<0.001$ ), thus IL-10 not reaching values of statistical significance. For our chosen Th1/Th2 immunological profile ratio of change (ratio IL-6/IL-10), we did not prove a statistically significant correlation with birth weight in the second trimester, but we have proved this correlation in the third trimester $\mathrm{p}<0.001$ (Table 4). To evaluate activation of cell-mediated immunity we chose absolute CD4(+) T cell count, we did not prove a statistically significant difference in the second and third trimester, between hypertension and reference group $(p=0.76 ; 0.42) \quad$ (Fig. 4). There were no statistically significant differences between hypertension and reference group in peripheral blood leukocytes levels in the second trimester $(p=0.18)$, while we found statistically significant difference in the third trimester $(p=0.04)$. There were no statistically significant correlations between the levels of uric acid UA and the levels of TNF- $\alpha$; IL-10; IL-6 (data not shown), we did not prove the correlation between uric acid UA and the risk of PE or birth weight value. Low birth weight (LBW) is 
Table 1. Clinical, immunological and metabolic characteristics of the reference and hypertension group at different time points.

\begin{tabular}{|c|c|c|c|c|}
\hline \multirow{2}{*}{ Characteristic } & \multicolumn{2}{|c|}{ Hypertension group $(n=20)$} & \multicolumn{2}{|c|}{ Reference group $(n=20)$} \\
\hline & Mean & Std. deviation & Mean & Std. deviation \\
\hline Age at delivery (years) & 33.55 & 2.67 & 34.10 & 3.24 \\
\hline \multicolumn{5}{|l|}{ Parity (\%) } \\
\hline Nulliparae & \multicolumn{2}{|c|}{52.6} & \multicolumn{2}{|c|}{50.1} \\
\hline Multiparae & \multicolumn{2}{|c|}{47.4} & \multicolumn{2}{|c|}{49.9} \\
\hline \multicolumn{5}{|l|}{ BMI at different time points $\left(\mathrm{kg} / \mathrm{m}^{2}\right)$} \\
\hline Trimester 2 & 28.14 & 4.76 & 28.72 & 5.23 \\
\hline Trimester 3 & 27.27 & 7.49 & 29.46 & 4.83 \\
\hline \multicolumn{5}{|c|}{ Cytokine levels at different time points } \\
\hline \multicolumn{5}{|c|}{$I L-6$} \\
\hline Trimester 2 & 2.74 & 2,96 & 0.5 & 0.0 \\
\hline Trimester 3 & 4.70 & 5.49 & 0.5 & 0.0 \\
\hline \multicolumn{5}{|l|}{$I L-10$} \\
\hline Trimester 2 & 3.04 & 3.91 & 0.57 & 0.28 \\
\hline Trimester 3 & 2.64 & 2.46 & 0.96 & 0.72 \\
\hline \multicolumn{5}{|l|}{$T N F-\alpha$} \\
\hline Trimester 2 & 2.65 & 1.02 & 2.00 & 0.00 \\
\hline Trimester 3 & 4.07 & 2.89 & 2.00 & 0.00 \\
\hline \multicolumn{5}{|c|}{ C-peptide at different time points $(\mu \mathrm{g} / \mathrm{l})$} \\
\hline Trimester 2 & 2.35 & 0.66 & 2.24 & 0.78 \\
\hline Trimester 3 & 1.60 & 0.32 & 2.83 & 1.11 \\
\hline \multicolumn{5}{|l|}{ HOMA-IR at different time points } \\
\hline Trimester 2 & 2.47 & 1.26 & 2.85 & 1.93 \\
\hline Trimester 3 & 2.04 & 1.05 & 2.70 & 1.59 \\
\hline \multicolumn{5}{|c|}{ Mean systolic blood pressure (SBP) at different time points } \\
\hline Trimester 2 & 143.98 & 5.15 & 117.08 & 5.25 \\
\hline Trimester 3 & 143.63 & 6.73 & 117.55 & 5.65 \\
\hline \multicolumn{5}{|c|}{ Mean diastolic blood pressure (DBP) at different time points } \\
\hline Trimester 2 & 89.25 & 2.84 & 70.00 & 4.00 \\
\hline Trimester 3 & 88.95 & 5.26 & 70.13 & 3.01 \\
\hline Preeclampsia cases (n) & & & & \\
\hline \multicolumn{5}{|l|}{ Newborns } \\
\hline Mean birth weight ( $g$ ) & $3,276.7$ & 278.2 & $3,506.0$ & 165.0 \\
\hline Week of delivery (week) & 38.75 & 1.70 & 39.25 & 0.77 \\
\hline Mean weight-for-age percentiles & 53.0 & 18.3 & 61.2 & 18.5 \\
\hline
\end{tabular}

Unless otherwise indicated, values are mean (SD) or median (IQR) (detectable); Values are presented as means \pm SD.

defined as a birth weight of less than 2,500 $\mathrm{g}$ (up to and including 2,499 g), as per the World Health Organization (Meikle et al. 2002). In our study, the minimum observed birth weight was $2,760 \mathrm{~g}$, thus none of the subjects met criteria for low birth weight. In addition levels of monitored cytokines TNF- $\alpha$; IL-10; IL- 6 in the second trimester; third trimester did not statistically significantly correlate with the week of delivery (Table 4). We chose a level of proinflammatory cytokine IL-6 and tested whether there is a statistically significant difference between the hypertension and reference group. Obvious and unambiguous differences are observed between the 
control and hypertension group. In the second trimester, $100 \%$ of the observed reference group had a low IL-6, while only $10 \%$ of the hypertension group had a low
IL-6 and $90 \%$ i.e. 18 had high IL-6 $(\mathrm{p}<0.001)$. In the third trimester, $100 \%$ of the hypertension group had high IL-6 and all the low's IL- 6 belong to the reference group.

Table 2. Comparison of the glycated hemoglobin (HbA1c) levels in reference group and gestational hypertension group at different time points.

\section{$\begin{array}{lll}\text { Characteristic } & \text { Hypertension group } & \text { Reference group }\end{array}$}

\section{Glycated hemoglobin (HbAlc)}

Trimester 2

$\begin{array}{lll}\text { Mean } & 35.05 & 34.60\end{array}$

Std. deviation

4.45

Difference (p-value)

0.78

Trimester 3

Mean

35.68

35.16

Std. deviation

3.92

Difference (p-value)

0.75

Values are expressed as mean $\pm \mathrm{SD}$, statistical significance is $p<0.05$

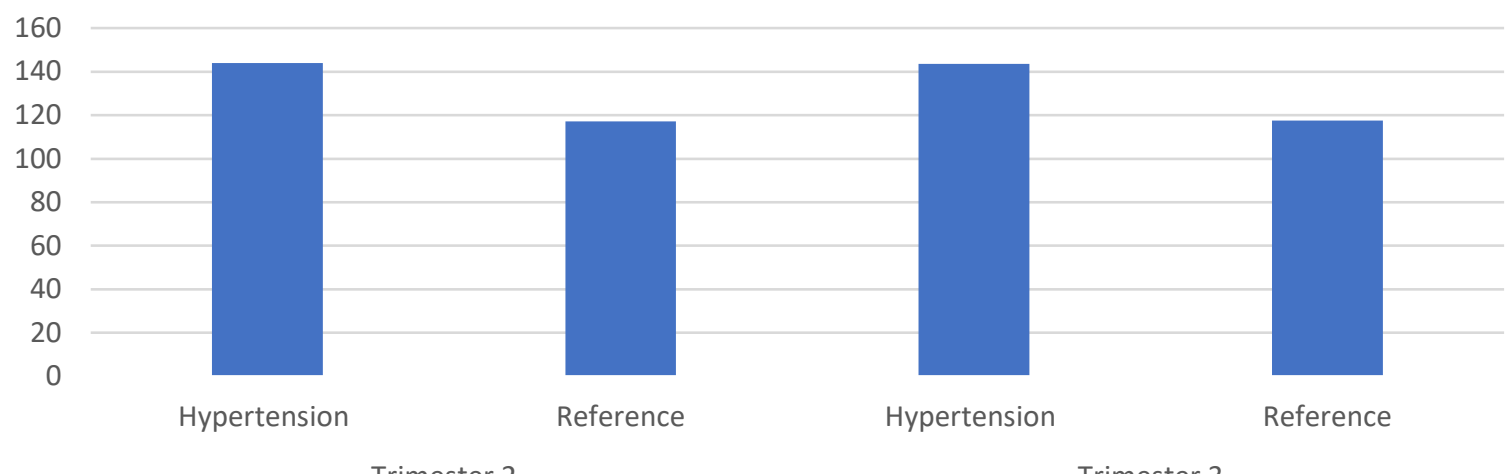

Trimester 2

Trimester 3

Fig. 1. Comparison of the SBP in reference group and gestational hypertension group at different time points. In the second and third trimester we proved a statistically significant difference in SBP between hypertension and reference group $(p<0.001 ; p<0.001)$. Statistical significance is from the Student t-test, values are expressed as mean $(\mathrm{mm} \mathrm{Hg})$.

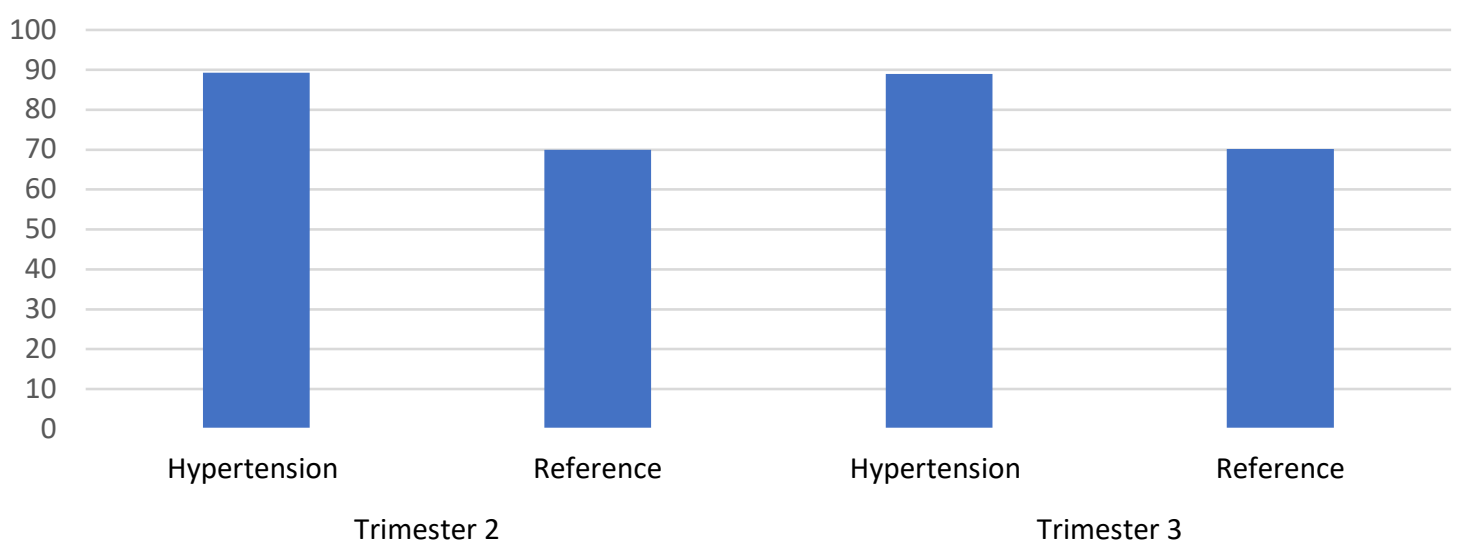

Fig. 2. Comparison of the DBP in reference group and gestational hypertension group at different time points. In the second and third trimester we proved a statistically significant difference in DBP between hypertension and reference group $(p<0.001 ; p<0.001)$. Statistical significance is from the Student t-test, values are expressed as mean $(\mathrm{mm} \mathrm{Hg})$. 
Table 3. The SBP parameters comparisons between group low and group high, subjects were divided into two groups according to their median of cytokine level (IL-6; TNF-a; IL-10): group low had a lower value than the median, group high had a higher value than median.

\begin{tabular}{|c|c|c|c|c|}
\hline \multirow{2}{*}{ Characteristic } & \multicolumn{2}{|c|}{ Trimester 2} & \multicolumn{2}{|c|}{ Trimester 3} \\
\hline & low IL-6 & high IL-6 & low IL-6 & high IL-6 \\
\hline Mean & 119.12 & 144.48 & 117.55 & 143.63 \\
\hline Std. deviation & 8.28 & 5.19 & 5.65 & 6.73 \\
\hline \multirow{2}{*}{$p$-value } & \multicolumn{2}{|c|}{$<0.001 \varphi \varphi$} & \multicolumn{2}{|c|}{$<0.001 \varphi \varphi$} \\
\hline & low TNF- $\alpha$ & high TNF- $\alpha$ & low TNF- $\alpha$ & high TNF- $\alpha$ \\
\hline Mean & 126.65 & 146.08 & 123.36 & 145.62 \\
\hline Std. deviation & 13.38 & 6.48 & 11.17 & 7.53 \\
\hline \multirow[t]{2}{*}{$p$-value } & \multicolumn{2}{|c|}{$<\mathbf{0 . 0 0 1} \varphi \varphi$} & \multicolumn{2}{|c|}{$<\mathbf{0 . 0 0 1} \varphi \varphi$} \\
\hline & low IL-10 & high IL-10 & low IL-10 & high IL-10 \\
\hline Mean & 123.19 & 144.17 & 120.31 & 136.76 \\
\hline Std. deviation & 11.67 & 8.13 & 7.14 & 14.47 \\
\hline p-value & \multicolumn{2}{|c|}{$<0.001 \varphi \varphi$} & \multicolumn{2}{|c|}{$0.0019 \varphi$} \\
\hline
\end{tabular}

Unless otherwise indicated, values are mean (SD) or median (IQR) (detectable). Statistical significance is from Kruskal-Wallis test. $\varphi \mathrm{P}<0.05, \varphi \varphi \mathrm{P}<0.01$.

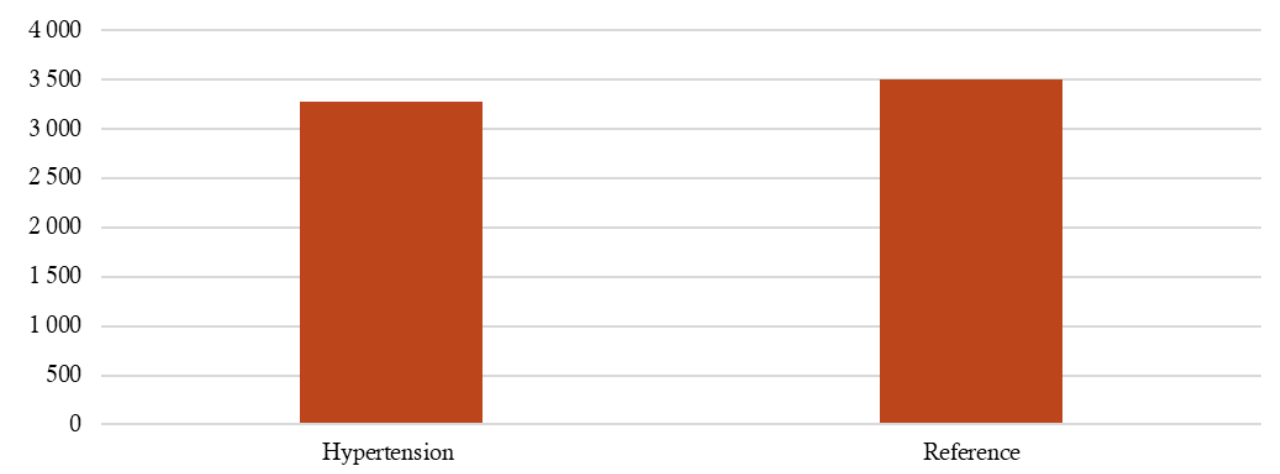

Fig. 3. Comparison of the birth weight in reference group and gestational hypertension group, we found the statistically significant difference in birth weight $(\mathrm{p}<0.05)$. Statistical significance is from the Student $t$-test, values are expressed as mean $(\mathrm{g})$.

Table 4. Spearman correlation coefficients of observed parameters (SBP; DBP), birth weight, week of delivery and cytokines (TNF-a, IL-6, IL-10) and UA among all subjects.

\begin{tabular}{lccccc}
\hline Characteristics & TNF- $\alpha$ & IL-10 & IL-6 & IL-6/IL-10 ratio & UA \\
\hline Trimester 2 & & & & & \\
Mean $S B P$ & $\mathbf{0 . 5 5} \varphi \varphi$ & $\mathbf{0 . 8 1} \varphi \varphi$ & $\mathbf{0 . 8 4} \varphi \varphi$ & 0.13 & -0.02 \\
Mean DBP & $\mathbf{0 . 5 2} \varphi \varphi$ & $\mathbf{0 . 7 6} \varphi \varphi$ & $\mathbf{0 . 7 9} \varphi \varphi$ & 0.11 & 0.01 \\
Birth weight $(g)$ & $\mathbf{- 0 . 3 2} \varphi$ & $\mathbf{- 0 . 5 1} \varphi \varphi$ & $\mathbf{- 0 . 5 6} \varphi \varphi$ & 0.07 & 0.27 \\
Week of delivery & -0.26 & -0.24 & -0.15 & 0.42 & -0.03 \\
\hline Trimester 3 & & & & & \\
Mean $S B P$ & $\mathbf{0 . 7 5} \varphi \varphi$ & $\mathbf{0 . 4 5} \varphi$ & $\mathbf{0 . 8 5} \varphi \varphi$ & $\mathbf{0 . 8 4} \varphi \varphi$ & 0.13 \\
Mean DBP & $\mathbf{0 . 7 0} \varphi \varphi$ & $\mathbf{0 . 4 9} \varphi$ & $\mathbf{0 . 8 7} \varphi \varphi$ & $\mathbf{0 . 8 6} \varphi \varphi$ & 0.11 \\
Birth weight $(g)$ & $\mathbf{- 0 . 5 3} \varphi$ & -0.27 & $\mathbf{- 0 . 5 1} \varphi \varphi$ & $\mathbf{- 0 . 5 8} \varphi \varphi$ & 0.12 \\
Week of delivery & -0.27 & -0.03 & -0.08 & -0.13 & -0.26 \\
\hline
\end{tabular}

Statistical significance is from the Spearman's rank correlation coefficient $\varphi p<0.05, \varphi \varphi p<0.01$. 
Table 5. The risk of preeclampsia parameters comparisons between group low and group high, subjects were divided into two groups according to their median of cytokine level (IL-6; TNF-a; IL-10): group low had a lower value than the median, group high had a higher value than median. Data are presented as relative frequencies (\%).

\begin{tabular}{|c|c|c|}
\hline Characteristic & low IL-6 & high IL-6 \\
\hline \multicolumn{3}{|l|}{ Trimester 3} \\
\hline Relative frequencies (\%) & 0 & 10 \\
\hline \multirow[t]{2}{*}{ p-value } & \multicolumn{2}{|c|}{0.15} \\
\hline & low TNF- $\alpha$ & high TNF- $\alpha$ \\
\hline Relative frequencies (\%) & 0 & 15.38 \\
\hline \multirow[t]{2}{*}{$p$-value } & \multicolumn{2}{|c|}{$0.04 \varphi$} \\
\hline & low IL-10 & high IL-10 \\
\hline Relative frequencies (\%) & 5 & 10 \\
\hline p-value & & \\
\hline
\end{tabular}

Statistical significance is from the Chi-square test, $\varphi p<0.05, \varphi \varphi p<0.01$.

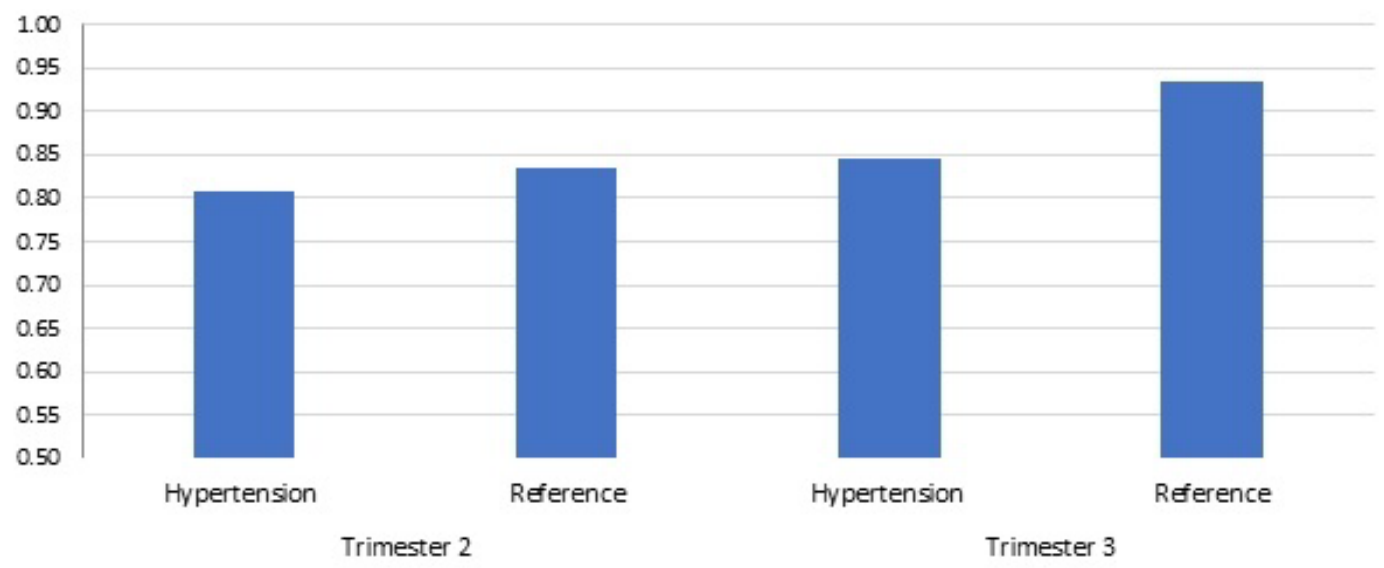

Fig. 4. Comparison of the absolute $\mathrm{CD} 4(+)$ count (peripheral blood) in reference group and gestational hypertension group at different time points, we did not prove a statistically significant difference in the second and third trimester, between hypertension and reference group $(p=0.76 ; 0.42)$. Statistical significance is from the Student $t$-test, values are expressed as $\times 10^{9} / / \mathrm{CD} 4(+)$.

\section{Discussion}

We assumed, that the levels of proinflammatory cytokines IL- 6 and TNF- $\alpha$ are an important part of the blood pressure control in pregnancy. This hypothesis, based on our acquired data, appears to be true. We have proved, that the mean SBP value is significantly higher for high IL-6 group, both in the second and third trimester $(p<0.001 ; p<0.001)$. Also, the elevation of mean SBP for high TNF- $\alpha$ group was statistically significant in both the second and third trimester $(\mathrm{p}<0.001 ; \mathrm{p}<0.001)$. There was also a proven statistically significant association between group low IL-10 and elevation of mean SBP in the second; third trimester $(\mathrm{p}<0.001 ; \mathrm{p}=0019)$. We found a significant positive correlation between TNF- $\alpha$; IL-6; IL-10 levels and SBP in the second trimester $(p<0.001$; $\mathrm{p}<0.001 ; \mathrm{p}<0.001)$; the third trimester $(\mathrm{p}<0.001 ; \mathrm{p}<0.001$; $\mathrm{p}<0.05)$. We also proved correlations for DBP during the second; third trimester $(\mathrm{p}<0.001 ; \mathrm{p}<0.001 ; \mathrm{p}<0.001)$; $(p<0.001 ; p<0.001 ; p<0.0015)$. Hence, our results support the hypothesis, that a proinflammatory state, (which in our opinion is significantly influenced by the elevated levels of proinflammatory cytokines IL-6 and TNF- $\alpha$ with a possible deficit of anti-inflammatory IL-10), is associated with higher levels of blood pressure.

Gestational diabetes and pregnancy-induced hypertension are common, and their relation is not well understood. A population-based case-control study was conducted using 1992-1998, consecutive cases of pregnancy-induced hypertension were divided into four: eclampsia, severe preeclampsia, mild preeclampsia, and gestational hypertension. Cases were compared with 
controls who did not have pregnancy-induced hypertension. Gestational diabetes was more common in each case group (3.9\% in eclamptics, $4.5 \%$ in severe preeclamptics, and $4.4 \%$ in both mild preeclamptics and those with gestational hypertension) than in controls $(2.7 \%)$. After adjustment gestational diabetes was associated with increased risk of severe preeclampsia odds ratio $(\mathrm{OR})=1.5$, mild preeclampsia $\mathrm{OR}=1.5$ and gestational hypertension $\mathrm{OR}=1.4$. $\mathrm{PE}$ is a pregnancyassociated disorder characterized by hypertension. In PE, cytokines such as TNF- $\alpha$ modify the expression of adhesion molecules in placental vessels, apoptosis of trophoblasts. Thus reduces trophoblast invasion and promotes placental ischemia. Defective placentation and decreased trophoblast invasion cause reduction in uteroplacental perfusion pressure (RUPP) and placental hypoxia. RUPP could stimulate the release of circulating bioactive factors, that cause imbalance with the pro-angiogenic factors vascular endothelial growth factor or cause the release of inflammatory cytokines, reactive oxygen species, hypoxia-induced factor-1. The circulating bioactive factors target endothelial cells causing generalized endothelial dysfunction. Decreased vasodilators and increased vasoconstrictors such as endothelin-1 and thromboxane A2, lead to increased vasoconstriction. Endothelin-1 (ET-1) is a major vasoconstrictor that may play a role in PE (Shah et al. 2015). Wallace et al. (2011) hypothesize that reduced uterine perfusion pressure (RUPP) induced CD4(+) $\mathrm{T}$ cells increase blood pressure during pregnancy. In pregnant rats circulating $\mathrm{TNF}-\alpha$ was elevated in recipients of RUPP CD4(+) T cells. These data support the hypothesis that TNF- $\alpha$ play an important role in the pathophysiology of hypertension in response to placental ischemia (Wallace et al. 2011). The importance of ET-1 in blood pressure control has been demonstrated on animal models, where an endothelin A and B receptor antagonists significantly increased the glomerular filtration rate, renal plasma flow and renal interstitial hydrostatic pressure (Kato et al. 1995). The correct function of endothelium seems to be the key for maintaining normal blood pressure, and it is during the ischemia of placenta that the number of M1-macrophages grows and the synthesis of proinflammatory cytokines TNF- $\alpha$, IL-1 $\beta$ and IL-18 increases. Those mentioned proinflammatory cytokines support the synthesis of highly effective vasoconstrictor endothelin and reduce the acetylcholine-induced vasodilation (Conrad et al. 1997). We emphasis, that endothelin, in animal models, decreased the excretion of sodium and the resulting decreased natriuresis is one of the main disorders participating in the development of hypertension (Kato et al. 1995).

A new lineage of T-helper (Th) cells that selectively produce IL-17 has been proposed. This population, which has been termed Th17, plays a critical role for the induction of inflammation. Activated dendritic cells increase production of proinflammatory cytokine IL-6 or IL-1, TGF-beta induced differentiation of native $\mathrm{T}$ cells diverted toward the Th17 cells pathway. The function of effector T cells, such as Th1, Th2 and Th17 cells, is regulated by $\mathrm{CD} 4(+) \mathrm{CD} 25(+)$ regulatory $\mathrm{T}$ (Treg) cells. (Sheu et al. 2018). We may indeed recall the hypothesis Wallace et al. (2011) that reduced RUPP induced $\mathrm{CD} 4(+) \mathrm{T}$ cells increase blood pressure during pregnancy. In our study we chose absolute CD4(+) T cell count to evaluate activation of cell-mediated immunity. We did not prove a statistically significant difference in absolute count of CD4(+) T cells in the second and third trimester between hypertension and reference group. We observed statistically significant difference in the third trimester $(p=0.04)$ in peripheral blood leukocytes levels, while we did not prove a significant difference in the second trimester $(\mathrm{p}=0.18)$ between hypertension and reference group. Our study had some limitations, which should be pointed out: design of our study was to assess change in circulating cytokine levels regarding to blood pressure, PE risk and low birth weight. We did not investigate the role of the Th subclasses in the induction of cytokine production and from the same reason it is also difficult to interpret the relation between absolute leukocyte count and observed parameters in the third trimester.

IL-6 is, as a proinflammatory cytokine, released by various cells: endothelial, smooth muscle cells, macrophages. The literature review shows that elevated levels of IL- 6 correlate with the elevation of blood pressure and are an independent risk factor for angiotensin II (Ang II) - induced hypertension (Ruiz et al. 2002). It was demonstrated in an animal model with induced hypertension (diet with increased servings of salt and distribution of Ang II infusion), that protein kinase B2 activates IL- 6 mediated inflammation. A deletion of protein kinase $\mathrm{B} 2$ in these models then normalized the blood pressure (Ruiz et al. 2002). Angiotensin II, on its own, potentiates synthesis of TNF- $\alpha$ and IL-6 (Zhang et al. 2003). Gadonski et al. (2006) conducted a series of experiments on rats, where by applying a clip they 
induced artificial reduction of uterine perfusion (RUPP) and demonstrated an increase of plasmatic levels of IL-6, significant increase of arterial pressure and reduction of glomerular filtration (eGF) in rats with RUPP compared to the control group. Endothelial dysfunction, which is associated with many forms of hypertension was, in human studies, at least partially mediated by the increased levels of proinflammatory cytokines (Saito et al. 2001). We conclude, that IL-6 and TNF- $\alpha$ are, according to human studies and animal models, significantly involved in the pathogenesis of insulin resistance and pregnancy hypertension. Our results demonstrated a statistically significant association between elevated levels of cytokines IL- 6 and TNF- $\alpha$ in the mother's peripheral blood and the elevation of SBP and DBP in GDM, a condition characterized by increased insulin resistance.

$\mathrm{PE}$ is diagnosed based on the presence of hypertension and proteinuria after the $20^{\text {th }}$ week of pregnancy in women that had normal blood pressure prior to gestation. PE is a multisystem disease characterized by endothelial dysfunction, increased peripheral vascular resistance, coagulation abnormalities, oxidative stress, hyperlipidemia and increased production of cytokines. PE is connected with spiral arteries remodeling impairment, defective cytotrophoblast invasion, a change in M1 and M2 macrophages ratio and subsequent progressive placental ischemia. This change is accompanied by an increase of proinflammatory cytokines (TNF- $\alpha$, IL-1 $\beta$ or IL-18) (Raghupathy et al. 2013). Many factors may be involved in further activation of monocytes during pre-eclampsia. Factors may be derived from the stressed placenta, such as anti-angiogenic factors, placental microparticles or ATP, which are released at increased amounts from the preeclamptic placenta (Redman et al. 2000). Previous research by Lockwood et al. (2008) demonstrated an elevated level of plasmatic IL-6 in women with PE and concluded, that decidua appears to be the source of IL-6 plasmatic elevation. Most likely through the mechanism of elevated levels of cytokines IL-1 and TNF- $\alpha$, leading to an increased expression of IL-6 mRNA by decidual cells. Experimental models in rats demonstrated connections between monocyte stimulation by TNF- $\alpha$ and elevation of nonclassical monocytes, increased inflammatory response, glomerular inflammation, hypertension and proteinuria, the state leading to preeclampsia (Lamarca et al. 2005). Several studies show an interesting association between cytokines, uric acid and PE. In women with PE, an elevated level of uric acid was described as early as the $10^{\text {th }}$ week of pregnancy (Powers et al. 2006). Our research results proved increased risk of $\mathrm{PE}$ in the third trimester only for group high TNF- $\alpha(p=0.04)$, groups high IL-6 and high IL-10 didn't meet the statistical significance criteria. In the second trimester, the increased risk of PE hasn't been statistically significant for any of the monitored cytokines (data not shown). The result of our research is fairly in accordance with the mentioned studies, we demonstrated a statistically significant association between mean SBP and levels of IL-6. If we bring back the hypothesis stated by Peraçolli et al. (2007) that increased production of cytokine TNF- $\alpha$ is a conjoint pathophysiological mechanism for gestational hypertension and $\mathrm{PE}$, where $\mathrm{PE}$ is just the most intensified version of this condition and understand, that elevation of blood pressure is a crucial part of PE syndrome, then we can't rule out, that the elevation of cytokine TNF- $\alpha$ is closely linked to the risk of developing $\mathrm{PE}$ and a larger sample of pregnant women would be needed in order to prove statistically significant associations with the risk of PE and with cytokine IL-6. If we consider the association between the risk of $\mathrm{PE}$ and TNF- $\alpha$ wasn't significant during the second trimester, but was significant in the third trimester, we regard this as likely, that significant elevation of TNF- $\alpha$ in the mother's peripheral blood is a matter of advanced syndrome. Our research results proved increased risk of $\mathrm{PE}$ in the third trimester only for group high TNF- $\alpha(p=0.04)$, better understanding of the association between cytokines and PE risk may lead to more effective strategies for prenatal care and may help to find a new way to prevent it. When assessing the correlation coefficient for birth weight during the second trimester, we proved statistically significant negative correlations for all the monitored cytokines TNF- $\alpha$; IL-10; IL-6 $\quad(\mathrm{p}<0.05 ; \quad \mathrm{p}<0.001$; $\mathrm{p}<0.001)$. Negative correlations were also proved in the third trimester $(p<0.05 ; p=0.096 ; p<0.001)$. Hence, in the third trimester, the correlation for IL-10 did not reach statistical significance. For the current literature, our results on correlation between cytokines and birth weight are rather contradictory. While our results proving a negative correlation between the levels of cytokine IL-6; TNF- $\alpha$ and birth weight remain consistent with prior research, our results for IL-10 are contradictory to literature sources. The study of Bartha et al. (2003) demonstrates an increased level of TNF- $\alpha$ in women with IUGR and placental insufficiency, but normal levels of TNF- $\alpha$ in women with IUGR and normal placental 
perfusion. The elevation of TNF- $\alpha$ was perceived as a phenomenon specific for IUGR with placental dysfunction. De Steenwinkel et al. (2013) provided evidence of association between elevation of IL-6 and low birth weight in mothers with rheumatoid arthritis. A majority of literature shows the protective effect of IL-10 on fetoplacetal unit (amongst other, by inhibition of secretion of proinflammatory cytokines IL-6, TNF- $\alpha$, interferon gamma) (Tinsley et al. 2010). A decreased level of IL-10 in the decidual region and trophoblast was described in pregnancies complicated by intrauterine growth restriction (IUGR) (Amu et al. 2006). On the contrary, we proved a negative correlation between birth weight and the levels of IL-10 in peripheral blood. Our study had some limitations. First, the biological effects of cytokines are determined by the interplay of cytokines and their soluble receptors. The levels of these soluble receptors were not determined. Secondly, it is known that body mass index may influence cytokine levels as well as pregnancy outcome and therefore it could be a potential confounder (Curry et al. 2008). Schmidt et al. (2015) found differences in pro- as well as anti-inflammatory cytokines when comparing obese and non-obese participants. Among the tested cytokines, IL-5, IL-10, IL-12, IL-13 and IFN- $\gamma$ were elevated in both general and central obesity. These findings do not indicate whether cytokines are a cause or a consequence of obesity, nor the underlying molecular mechanisms (Schmidt et al. 2015). In another study serum concentrations of CRP, TNF- $\alpha$ and IL-6 were significantly correlated with weight, BMI, waist circumference, hip circumference and waist-hip ratio (Park et al. 2005). In our study reference group was chosen to fit the characteristics of the hypertension group in both age, and BMI. We observed greater increase in BMI for the reference group compared to hypertension group in the third trimester. These findings support our hypothesis that proinflammatory cytokines play a role in regulating blood pressure and observed elevated levels of IL-6 and TNF- $\alpha$ are not related to changes in BMI over the third trimester. Despite the mentioned limitation of our study, we consider our findings of elevated levels of proinflammatory cytokines IL-6, TNF- $\alpha$ in relation to SBP and DBP in mothers with GDM inspiring and an interesting topic for future research.

\section{Conclusions}

We found a significant positive correlation between TNF- $\alpha$; IL-6; IL-10 levels and SBP in the second trimester $(\mathrm{p}<0.001 ; \mathrm{p}<0.001 ; \mathrm{p}<0.001)$; the third trimester $(\mathrm{p}<0.001 ; \mathrm{p}<0.001 ; \mathrm{p}<0.05)$. We also proved correlations for DBP during the second; third trimester $(\mathrm{p}<0.001 ; \quad \mathrm{p}<0.001 ; \quad \mathrm{p}<0.001) ; \quad(\mathrm{p}<0.001 ; \quad \mathrm{p}<0.001 ;$ $\mathrm{p}<0.0015)$. Furthermore, we proved a statistically significant higher risk of PE for high TNF- $\alpha$ group in the third trimester $(p=0.04)$. The evaluation of correlation coefficient for birth weight during the second trimester yielded statistically significant negative correlations for TNF- $\alpha$; IL-10; IL-6 p-value $(\mathrm{p}<0.05 ; \mathrm{p}<0.001 ; \mathrm{p}<0.001)$. Prediction of $\mathrm{PE}$ is widely accepted to rely on multiparametric approaches because no single screening test provides a reasonable risk assessment. The screening of cytokine levels (TNF- $\alpha$; IL-10; IL-6) during the second trimester might be premature from the clinical point of view, cost-benefit analysis performed in large clinical studies are needed. In our study, the minimum observed birth weight was $2,760 \mathrm{~g}$. Therefore none of the subjects met the criteria for low birth weight. The results of our study confirm the hypothesis, that the proinflammatory state, together with the elevation of proinflammatory TNF- $\alpha$; IL-6 cytokines, play an important role in the elevation of SBP and DBP. Moreover, we have found negative correlation between TNF- $\alpha$; IL- 6 and birth weight. Our results are in accordance with the literature (De Steenwinkel et al. 2013), which shows that the levels of maternal cytokines can significantly influence the growth of the fetus in women with rheumatoid arthritis. Our results confirmed a similar effect of proinflammatory cytokines in women with GDM. Further studies are undoubtedly needed to clarify the association of maternal cytokine productions and pregnancy complications

\section{Conflict of Interest}

There is no conflict of interest.

\section{Acknowledgements}

The study was supported by the educational grant Second Department of Internal Medicine, Faculty of Medicine, Masaryk University. The authors are grateful for essential help with paper: statistical analysis and advice on the statistical presentation Matej Marek, laboratory staff Department of Clinical Immunology and Allergology St. Anne's University Hospital in Brno and especially study nurse Jana Havrankova. 


\section{References}

AMERICAN DIABETES ASSOCIATION: 13. Management of Diabetes in Pregnancy: Standards of Medical Care in Diabetes-2018. Diabetes Care 41 (Suppl 1): S137-S143, 2018.

AMU S, HAHN-ZORIC M, MALIK A, ASHRAF R, ZAMAN S, KJELLMER I, HAGBERG H, PADYUKOV L, HANSON LA: Cytokines in the placenta of Pakistani newborns with and without intrauterine growth retardation. Pediatr Res 59: 254-258, 2006.

AZIZIEH F, RAGHUPATH R: Tumor necrosis factor- $\alpha$ and pregnancy complications: A prospective study. Med Princ Pract 24: 165-170, 2015.

BARTHA JL, ROMERO-CARMONA R, COMINO-DELGADO R: Inflammatory cytokines in intrauterine growth retardation. Acta Obstet Gynecol Scand 82: 1099-1102, 2003.

CHALLIS JR, LOCKWOOD CJ, MYATT L, NORMAN JE, STRAUSS JF 3RD, PETRAGLIA F: Inflammation and pregnancy. Reprod Sci 16: 206-215, 2009.

CHAOUAT G, LEDÉE-BATAILLEA N, DUBANCHETA S, ZOURBASA S, SANDRAC O, MARTAL J: TH1/TH2 paradigm in pregnancy: paradigm lost? Cytokines in pregnancy/early abortion: reexamining the TH1/TH2 paradigm. Int Arch Allergy Immunol 134: 93-119, 2004.

CONRAD KP, BENYO DF: Placental cytokines and the pathogenesis of preeclampsia. Am J Reprod Immunol 37: 240-249, 1997.

CURRY AE, VOGEL I, SKOGSTRAND K: Maternal plasma cytokines in early-and mid-gestation of normal human pregnancy and their association with maternal factors. $J$ Reprod Immunol 77: 152-160, 2008.

CUTLAND C, LACKRITZ E, MALLETT T, MOORE E, BARDAJÍ A, CHANDRASEKARAN R, LAHARIYA CH, NISAR H, MILAGRITOS I, TAPIA J, PATHIRANA J, KOCHHAR S, MUÑOZ F, THE BRIGHTON COLLABORATION LOW BIRTH WEIGHT WORKING GROUP: LOW BIRTH WEIGHT: Case definition $\&$ guidelines for data collection, analysis, and presentation of maternal immunization safety data. Vaccine $\mathbf{3 5}$ : 6492-6500, 2017.

DE STEENWINKEL FD, HOKKEN-KOELEGA AC, DE MAN YA, DE RIJKE YB, DE RIDDER MA, HAZES JM: Circulating maternal cytokines influence fetal growth in pregnant women with rheumatoid arthritis. Ann Rheum Dis 72: 1995-2001, 2013.

FERNANDEZ-VOJVODICH P, ZAMAN F, SÄVENDAHL L: Interleukin-6 acts locally on the growth plate to impair bone growth. Ann Rheum Dis 72: 24, 2013.

GADONSKI G, LAMARCA BB, SULLIVAN E, BENNETT W, CHANDLER D, GRANGER JP: Hypertension produced by reductions in uterine perfusion in the pregnant rat role of interleukin 6. Hypertension 48: 711-716, 2006.

GOMES CP, TORLONI MR, GUEUVOGHLANIAN-SILVA BY, ALEXANDRE SM, MATTAR R, DAHER S: Cytokine levels in gestational diabetes mellitus: A systematic review of the literature. Am J Reprod Immunol 69: 545-557, 2013.

HENNESSY A, PILMORE HL, SIMMONS LA, PAINTER DM: A deficiency of placental IL-10 in preeclampsia. J Immunol 163: 3491-3495, 1999.

KATO T, KASSAB S, WILKINS FC, KIRCHNER K, KEISER J, GRANGER JP: Endothelin antagonist improve renal function in spontaneously hypertensive rats. Hypertension 25: 883-887, 1995.

KIRWAN JP, HAUGUEL-DE MOUZON S, LEPERCQ J, CHALLIER JC, HUSTON-PRESLEY L, FRIEDMAN JE, KALHAN SC, CATALANO PM: TNF-alpha is a predictor of insulin resistance in human pregnancy. Diabetes 51: 2207-2213, 2002.

KOUREMBANAS S, MCQUILLAN LP, LEUNG GK, FALLER DV: Nitric oxide regulates the expression of vasoconstrictors and growth factors by vascular endothelium under both normoxia and hypoxia. $J$ Clin Invest 92: 99-104, 1993.

LAMARCA BB, BENNETT WA, ALEXANDER BT, COCKRELL K, GRANGER JP: Hypertension produced by reductions in uterine perfusion in the pregnant rat. Role of tumor necrosis factor. Hypertension 46: 1022-1025, 2005.

LANA K, WAGNER MD: First Choice Community Healthcare, Albuquerque, New Mexico. 
LASH GE, ERNERUDH J: Decidual cytokines and pregnancy complications: focus on spontaneous miscarriage. J Reprod Immunol 108: 83-89, 2015.

LOCKWOOD CJ, YEN CF, BASAR M, KAYISLI UA, MARTEL M, BUHIMSCHI I, BUHIMSCHI C, HUANG SJ, KRIKUN G, SCHATZ F: Preeclampsia-related inflammatory cytokines regulate interleukin-6 expression in human decidual cells Am J Pathol 172: 1571-1579, 2008.

MATSUDA M, DEFRONZO R: Insulin sensitivity indices obtained from oral glucose tolerance testing. Diabetes Care 22: 1462-1470, 1999.

MATTHEWS D, HOSKER J, RUDENSKI A, NAYLOR B, TREACHER D, TURNER R: Homeostasis model assessment: insulin resistance and b-cell function from fasting plasma glucose and insulin concentrations in man. Diabetologia 28: 412-419, 1985.

MEIKLE S, FERRE C, PETERSON H, JENG G, WILCOX L: Low and very low birth weight in infants conceived with use of assisted reproductive technology. $N$ Engl J Med 346: 731-737, 2002.

ORGANIZATION WH. International statistical classification of diseases and related health problems, tenth revision, 2nd ed. World Health Organization. 94-99, 2004.

PARK HS, PARK JY, YU R: Relationship of obesity and visceral adiposity with serum concentrations of CRP, TNF-alpha and IL-6. Diabetes Res Clin Pract 69: 29-35, 2005.

PERAÇOLI JC, RUDGE MV, PERAÇOLI M: Tumor necrosis factor-alpha in gestation and puerperium of women with gestational hypertension and pre-eclampsia. Am J Reprod Immunol 57: 177-185, 2007.

POWERS RW, BODNAR LM, NESS RB: Uric acid concentrations in early pregnancy among preeclamptic women with gestational hyperuricemia at delivery. Am J Obstet Gynecol 194: 160, 2006.

QUIÑONES GALVAN A, NATALI A, BALDI S, FRASCERRA S, SANNA G, CIOCIARO D, FERRANNINI E: Effect of insulin on uric acid excretion in humans. Am J Physiol 268: E1-E5, 1995.

RAGHUPATHY RAJ: Cytokines as key players in the pathophysiology of preeclampsia. Med Princ Pract 22: 8-19, 2013.

REDMAN CW, SARGENT IL: Placental debris, oxidative stress and pre-eclampsia. Placenta 21: 597-602, 2000.

RUIZ-ORTEGA M, RUPEREZ M, LORENZO O, ESTEBAN V, BLANCO J, MEZZANO S, EGIDO J: Angiotensin II regulates the synthesis of proinflammatory cytokines and chemokines in the kidney. Kidney Int 82: 12-22, 2002.

SAITO S: Cytokine network at the feto-maternal interface. J Reprod Immunol 47: 87-10, 2001.

SANTNER-NANAN B, PEEK MJ, KHANAM R, RICHARTS L, ZHU E, FAZEKAS DE ST GROTH B, NANAN R: Systemic increase in the ratio between Foxp3+ and IL-17-producing CD4+ T cells in healthy pregnancy but not in preeclampsia. J Immunol 183: 7023-7030, 2009.

SCHMIDT FM, WESCHENFELDER J, SANDER C, MINKWITZ J, THORMANN J, CHITTKA T, MERGL R, KIRKBY KC, FAßHAUER M, STUMVOLL M, HOLDT LM, TEUPSER D, HEGERL U, HIMMERICH H: Inflammatory cytokines in general and central obesity and modulating effects of physical activity. PLoS One 10: $\mathrm{e} 0121971,2015$.

SHAH D, KHALIL R: bioactive factors in uteroplacental and systemic circulation link placental ischemia to generalized vascular dysfunction in hypertensive pregnancy and preeclampsia. Biochem Pharmacol 15: 211-226, 2015.

SHEU A, CHAN Y, FERGUSON A, BAKHTYARI MB, HAWKE W, WHITE C, CHAN YF, BERTOLINO PJ, WOON HG, PALENDIRA U, SIERRO F, LAU SM: A proinflammatory $\mathrm{CD}^{+}{ }^{+} \mathrm{T}$ cell phenotype in gestational diabetes mellitus. Diabetologia 61: 1633-1643, 2018.

TAYLOR RN, VARMA M, TENG NN, ROBERTS JM: Women with preeclampsia have higher plasma endothelin levels than women with normal pregnancies. J Clin Endocrinol Metab 71: 1675-1677, 1990.

TINSLEY JH, SOUTH S, CHIASSON VL, MITCHELL BM: Interleukin-10 reduces inflammation, endothelial dysfunction, and blood pressure in hypertensive pregnant rats. Am J Physiol Regul Integr Comp Physiol 298: R713-R719, 2010.

WAGNER L: Diagnosis and management of preeclampsia. Am Fam Physician 70: 2317-2324, 2004. 
WALLACE K, RICHARDS S, DHILLON P, WEIMER A, EDHOLM ES, BENGTEN E: CD4+ T-helper cells stimulated in response to placental ischemia mediate hypertension during pregnancy. Hypertension 57: 949-955, 2011.

WANG Y, WALSH SW: TNF alpha concentrations and mRNA expression are increased in preeclamptic placentas. J Reprod Immunol 32: 157-169, 1996.

ZHANG ZH, WEI SG, FRANCIS J, FELDER RB: Cardiovascular and renal sympathetic activation by blood-borne TNF in rat: the role of central prostaglandins. Am J Physiol Regul Integr Comp Physiol 284: R916-R927, 2003. 